Reprinted with permission from http://dx.doi.org/10.1063/1.3686349, Copyright 2011, American Institute of Physics.

This article may be downloaded for personal use only.

Any other use requires prior permission of the author and the American Institute of Physics.

\title{
ANFO RESPONSE TO LOW-STRESS PLANAR IMPACTS
}

\author{
M. A. Cooper*, W. M. Trott*, R. G. Schmitt*, M. Short ${ }^{\dagger}$ and S. I. Jackson ${ }^{\dagger}$ \\ * Sandia National Laboratories, Albuquerque, NM, 87185, USA \\ ${ }^{\dagger}$ Los Alamos National Laboratory, Shock \& Detonation Physics, Los Alamos, NM 87544, USA
}

\begin{abstract}
Ammonium Nitrate plus Fuel Oil (ANFO) is a non-ideal explosive where the mixing behavior of the mm-diameter prills with the absorbed fuel oil is of critical importance for chemical energy release. The large-scale heterogeneity of ANFO establishes conditions uniquely suited for observation using the spatially- and temporally-resolved line-imaging ORVIS (Optically Recording Velocity Interferometer System) diagnostic. The first demonstration of transmitted wave profiles in ANFO from planar impacts using a single-stage gas gun is reported. Major observations including an extended compaction precursor, post-shock particle velocity variations and between-prill jetting are reported.
\end{abstract}

Keywords: ORVIS, interferometry, impact: velocity.

PACS: 62.50.Ef, 07.60.Ly.

\section{INTRODUCTION}

This work supports development of predictive tools aimed at the performance of non-ideal explosives $[1,2,3]$. In particular, ammonium nitrate plus fuel oil (ANFO) is one such non-ideal, heterogeneous explosive with two mean characteristic length scales, namely that of the in-prill porosity and the mean prill diameter $[1,2]$. The prill-to-prill interactions and prill compaction that occur under shock loading establish the conditions necessary for initiating chemical reaction between the ammonium nitrate (AN) and fuel oil (FO). Observations of the ANFO response to impacts at low-stress and near detonation conditions were conducted in a gas gun with a coupled optically recording velocity interferometer system (ORVIS) capable of collecting spatiallyand temporally-resolved particle velocity measurements. This work presents initial experimental data, discusses the main observations and future plans.

\section{EXPERIMENTAL}

The experiments were conducted on the single stage gas gun at SNL's Explosive Components Facil- ity that has an 18 -meter long and a 6.35 -cm diameter barrel. Impact velocities range from $0.17-1.40 \mathrm{~km} / \mathrm{s}$. Figure 1 presents the experimental arrangement of the ORVIS system coupled to the gas gun, e.g., see $[3,4]$. In short, interference fringes from the ORVIS diagnostic is recorded on a streak camera where motion at the target is recorded as fringe displacement. Analysis of the collected raw image data follows a "rolling push-pull" method $[4,5]$.

The target assembly consists of a Kel-F sample cup surrounded by a target ring used for mounting to the end of the gas gun barrel (Fig. 2). Explosive grade AN from Dyno Nobel having mean particle sizes between 1.4-2.0 $\mathrm{mm}$ [6] were mixed with diesel fuel oil in a ratio of $96 \%$ wt $\mathrm{AN}$ and $4 \%$ wt FO. The total net explosive weight in each gas gun target was nominally 20.15 grams. The AN and FO were mixed by shaking a small plastic vial for 10 minutes then poured into the Kel-F sample cup and lightly tamped (left photo of plastic vial for 10 minutes then poured into the Kel-F sample cup and lightly tamped (left photo of Fig. 3). A PMMA window containing a $12.7-\mu \mathrm{m}$-thick aluminum foil backed by a 0.229 -mm-thick Kapton buffer was lightly pressed against the ANFO surface (right photo of Fig. 3). The 


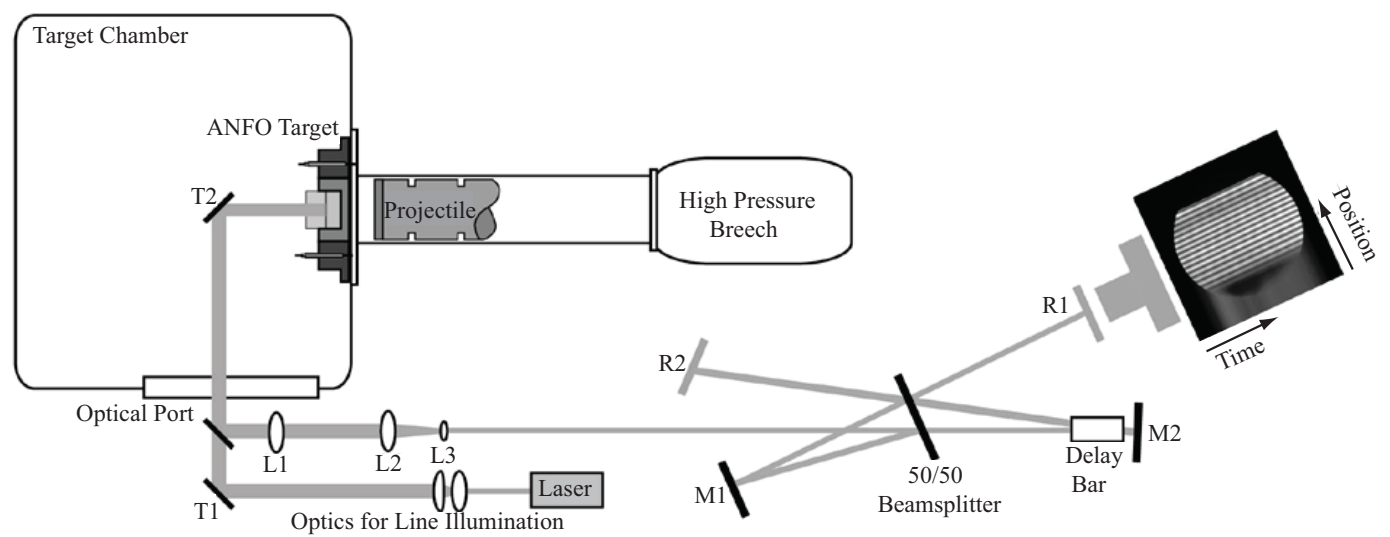

FIGURE 1. Illustration of ORVIS system coupled to an ANFO-containing gas gun target.

window was held in place with set screws through the Kel-F sample cup and sealed with 5-minute epoxy.

The internal Kel-F sample cup diameter is 40.6 $\mathrm{mm}$ (20-29 prill dia.) and the ANFO bed thickness is $18.7 \mathrm{~mm}$ (9-13 prill dia.) for a final measured density of $0.83-0.84 \mathrm{~g} / \mathrm{cc}$. The ORVIS laser line illuminating the target is approximately $12 \mathrm{~mm}$ long (6-8 prill dia.) resulting in an image magnification at the streak camera image plane of 1.4. The fringe spacing on the target was nominally $485 \mu \mathrm{m}(0.25-0.35$ prill dia. $)$. All of the raw data images record a $5 \mu$ s streak time.

The next sections present the raw image data and reduced particle velocity data collected from three tests at varying stress conditions within the ANFO. The stress values are calculated using impedance matching techniques from the measured impact velocity and the unreacted porous ammonium nitrate Hugoniot, $\rho=0.86 \mathrm{~g} / \mathrm{cc}, U_{s}=0.84+1.42 u_{p}$ [7].

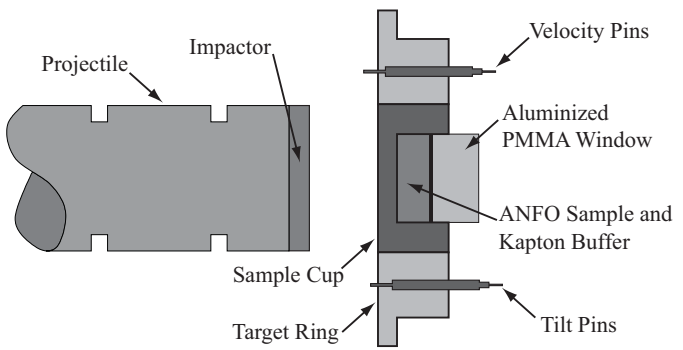

FIGURE 2. Illustration of wedge target and projectile.

\section{LOW STRESS CONDITIONS WITH P $=1.81$ GPA}

Figure 4 presents raw image data for the low-stress condition. The projectile with an aluminum impactor impacted the Kel-F sample cup at a velocity of 0.92 $\mathrm{km} / \mathrm{s}$. A precursor compaction wave appears at the aluminized window after $1.6 \mu$ s from the start of the streak image. The duration of this compaction wave persists in time until a dramatic loss of intensity of the diffuse return laser light at approximately $3.2 \mu \mathrm{s}$ from the start of the streak image. A spatial variation in this loss of intensity is observed corresponding to the width of approximately 3-4 prill diameters. The reason for this dramatic loss of light intensity is not precisely known in these ANFO tests. However, typical reasons for loss of return light intensity can be attributed to the loss of the aluminized observation surface (by ablation or melting when subjected to hightemperature shock conditions) or non-planar defor-

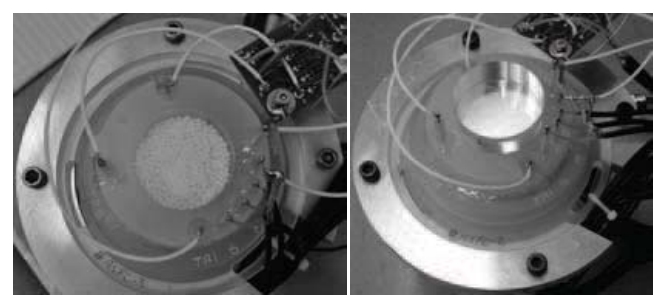

FIGURE 3. Photo of target showing ANFO prills in Kel-F sample cup (left) and aluminized PMMA window installed (right). 


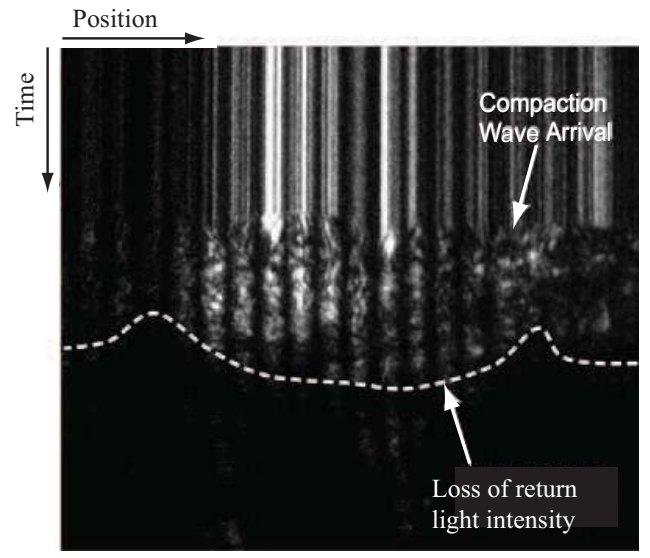

FIGURE 4. Raw image data for the low stress condition. The image has been cropped from its original size.

mation of the aluminized observation surface (thus, directing the reflected laser light out of the collection volume of L1 in Fig. 1). It is speculated that under these relatively low stress conditions and highly heterogeneous ANFO material, the non-planar deformation of the aluminized observation surface is the most likely cause of return light intensity loss. Near the center of the image, several fringes are still observable and show a gradual displacement indicating a continuous increase in particle velocity.

The particle velocities associated with the fringes with persistent contrast during the entire record were processed into particle velocities and plotted in Fig. 5. As in the raw data, significant particle velocity oscillations are present within the compaction wave

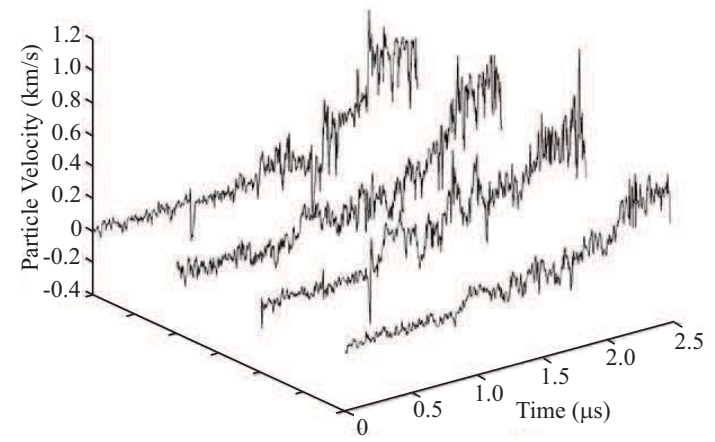

FIGURE 5. Processed particle velocity data for four selected fringes of Fig. 4.

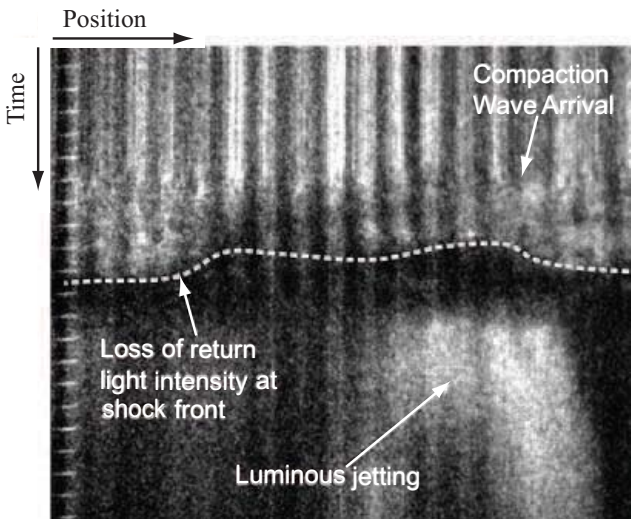

FIGURE 6. Raw image data for the intermediate stress condition. The image has been cropped from its original size.

and during the gradual particle velocity acceleration during the dispersive shock wave. With our ORVIS diagnostic typical uncertainties in the velocity are less than $1 \%$. For these tests, a complete uncertainty analysis is a subject of future work after further experimental improvements are made to improve the signal-to-noise ratio of the raw image data and the dramatic loss of return light intensity.

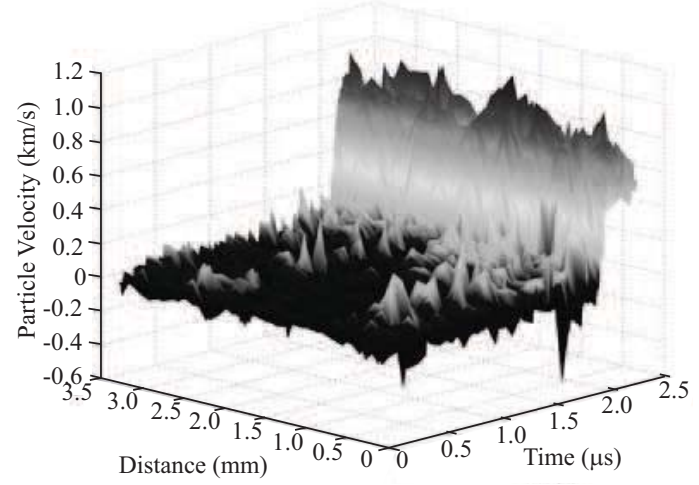

FIGURE 7. Surface plot of processed particle velocity data as a function of time and position for the raw fringe data of Fig. 6. 
Reprinted with permission from http://dx.doi.org/10.1063/1.3686349, Copyright 2011, American Institute of Physics.

This article may be downloaded for personal use only.

Any other use requires prior permission of the author and the American Institute of Physics.

\section{INTERMEDIATE STRESS CONDITIONS WITH P = 2.34 GPA}

Figure 6 presents the raw image data for an intermediate stress condition. The projectile with an aluminum impactor impacted the Kel-F sample cup at a velocity of $1.10 \mathrm{~km} / \mathrm{s}$. As in the low-stress condition, the arrival of the precursor compaction is observed followed by a loss of diffuse return light intensity. At this condition, the shock is less dispersive and is followed by distinct regions of luminous broadband intensity indicative of between-prill jetting. This broadband light is distinctly different than the regular structure of the raw fringes such that the luminosity must be from a chemiluminescent source (chemical reaction in the ANFO or burning of the aluminized observation surface) rather than due to the interference of the ORVIS laser light.

The raw image data was processed to create a surface plot of particle velocity as a function of time and position (Fig. 7). The center $4 \mathrm{~mm}$ of the raw image data is analyzed. As in the raw data, the arrival of the low level compaction wave followed by a less-dispersive shock to the post-shock condition is shown. The significant spatial and temporal particle velocity oscillations are clearly a consistent characteristic of this heterogeneous material over a range of stress wave conditions.

\section{NEAR DETONATION CONDITIONS WITH P = 4.04 GPA}

Figure 8 presents the raw image data for a neardetonation stress condition. The projectile with a stainless steel 304 impactor impacted the Kel-F sample cup at a velocity $1.3 \mathrm{~km} / \mathrm{s}$. In this test, the streak

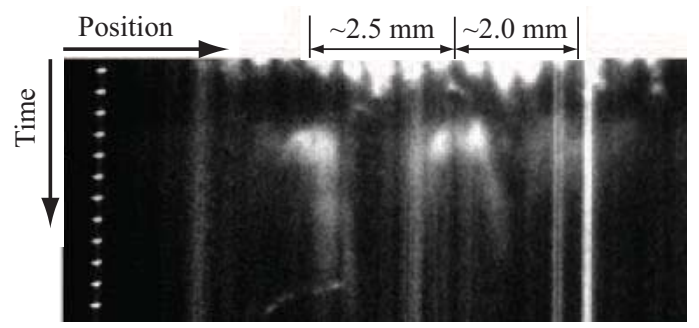

FIGURE 8. Raw image data for the near-detonation stress condition. The image has been cropped from its original size. image timing missed the arrival of the precursor compaction wave but did capture the point where the loss of return light intensity occurred. Here, the scalloped behavior of the wave is noted with a period on the order of $2.0-2.5 \mathrm{~mm}$ or 1-2 prill diameters. Also observed is the luminous jetting that appears to coincide with the wave shape. This prill-dependent behavior has also been observed in overdriven detonation experiments using the PDV diagnostic [8].

\section{CONCLUSIONS}

We have utilized our ORVIS diagnostic and gas gun facility to collect data on the response of ANFO to low-stress planar impacts. Many features are observed in these preliminary results including a prolonged compaction precursor, loss of return light intensity at shock arrival, and luminous between-prill jetting. Ongoing efforts are focused at improving buffer-window setup to prolong data collection and quantifying shock dispersion and spatial/temporal frequencies of particle velocity oscillations.

\section{ACKNOWLEDGEMENTS}

Sandia National Laboratories is a multiprogram laboratory managed and operated by Sandia Corporation, a wholly owned subsidiary of Lockheed Martin Company, for the U.S. Department of Energy's National Nuclear Security Administration under Contract DE-AC04-94AL85000. Approved for public release, LA-UR 11-04325.

\section{REFERENCES}

1. Baer, M. R., Gartling, D. K., and DesJardin, P. E., "Combustion theory and modeling," Accepted 2011.

2. Short, M., Los Alamos National Laboratory Tech. Rep. LA-UR 09-00362 (2009).

3. Trott, W.M., et al., J. Appl. Phys. 101, 024917 (2007).

4. Trott, W. M., Knudson, M. D., Chhabildas, L. C., and Asay, J. R., AIP Conf. Proc. 505, 993-998 (2000).

5. Trott, W. M., et al., Fundamental Issues and Applications of Shock-Wave and High-Strain-Rate Phenomena, (K. Staudhammer, et al. eds.), 647-654 (2001).

6. www.dynonobel.com.

7. Dremin, A. N., Shvedov, K. K., and Avdonin, O. S., Combust. Explo. Shock 6, 449-455 (1970).

8. Jensen, B., and Short, M. "ANFO detonation reaction zone structure via $\mathrm{Cu}$ flyer plate impact," personal communication (2011). 